\title{
Six year experience of transvenous left ventricular lead implantation for permanent biventricular pacing in patients with advanced heart failure: technical aspects
}

\author{
C Alonso, C Leclercq, F Revault d'Allonnes, D Pavin, F Victor, P Mabo, J-C Daubert
}

\begin{abstract}
Background-Biventricular pacing has been proposed as an adjuvant to optimal medical treatment in patients with drug refractory heart failure caused by chronic left ventricular systolic dysfunction and intraventricular conduction delay.

Objective-To assess the technical feasibility and long term results (over six years) of transverse left ventricular pacing with the lead inserted into a tributary vein of the coronary sinus.

Subjects-From August 1994 to February 2000, left ventricular lead implantation was attempted in 116 patients who were eligible for biventricular pacing (mean (SD) age 67 (9) years, New York Heart Association (NYHA) functional class III/IV, left ventricular ejection fraction 22 (6)\%, QRS duration 185 (26) $\mathrm{ms}$ ).

Results-The overall implantation success rate was $88 \%(\mathrm{n}=102)$. A learning curve was indicated by a progressive increase in success from $61 \%$ early on to $98 \%$ in the last year. The mean pacing threshold was $1.1(0.7) \mathrm{V} / 0.5 \mathrm{~ms}$ at the time of implantation and increased slightly up to 1.9 (0.9) V/0.5 ms at the end of the follow up period (15 (13) months). The rate of acute and delayed left ventricular lead dislodgement decreased from $30 \%$ in the early years to $11 \%$ after 1999 . During follow up, 19 patients required reoperation for delayed lead dislodgement or increase in left ventricular pacing threshold $(n=15)$, phrenic nerve stimulation $(n=3)$, or infection $(n=3)$.

Conclusions-Transverse left ventricular pacing through the coronary sinus is feasible and safe. The rate of implantation failure and of lead related problems has decreased greatly with increasing experience and with improvements in the equipment.

(Heart 2001;86:405-410)
\end{abstract}

Keywords: biventricular pacing; left ventricular pacing; heart failure; intraventricular conduction delay

Biventricular pacing was proposed in 1994 as an adjuvant to medical treatment in patients with drug refractory heart failure caused by chronic left ventricular systolic dysfunction and intraventricular delay. ${ }^{12}$ Following encouraging results from acute haemodynamic studies, ${ }^{3-6}$ several groups initiated pilot studies of permanent biventricular pacing. ${ }^{78}$ Results from those open, non-randomised trials were encouraging, revealing a significant improvement in New York Heart Association (NYHA) functional classification, exercise tolerance, and quality of life in those patients. One of the technical difficulties of this novel therapeutic approach is to achieve effective, safe, and permanent pacing of the left ventricle. Three methods have been proposed for implanting the left ventricular pacing lead. Early implantations were performed by the epicardial route, ${ }^{12}$ but this method requires a thoracotomy or thoracoscopy under general anaesthesia, and the complication rate is too high. Second, certain groups have proposed pacing the left ventricular endocardially. With this technique, a lead is placed in the left ventricle after trans-septal catheterisation. ${ }^{9}$ The technique is still being evaluated and is currently reserved for use in cases of coronary sinus implantation failure. The third method is coronary sinus implantation, initially proposed by Daubert, and this is currently the most popular. ${ }^{10}$ Its main advantage is a totally transvenous approach.
The lead, which is inserted into the subclavian vein, is positioned in a tributary of the coronary sinus. However, the difficulties posed by this method are not negligible in view of the pronounced anatomical variation in coronary sinus anatomy from patient to patient.

We report a six year experience of biventricular pacing, where the left ventricle was paced by a lead inserted into a branch of the coronary sinus.

\section{Methods}

INCLUSION CRITERIA

All patients at our centre with consecutively implanted biventricular pacemakers were included in the study. All met the following criteria:

- severe heart failure (NYHA class III or IV) despite optimised medical treatment, featuring at least angiotensin converting enzyme (ACE) inhibitors or angiotensin receptor blockers, diuretics, and more recently $\beta$ blockers;

- heart failure was related to chronic left ventricular systolic dysfunction, regardless of aetiology, with a left ventricular end diastolic diameter of $>60 \mathrm{~mm}$ and a left ventricular ejection fraction of $<35 \%$;

- all had major intraventricular conduction delay, with an intrinsic QRS duration of $>150 \mathrm{~ms}$ 


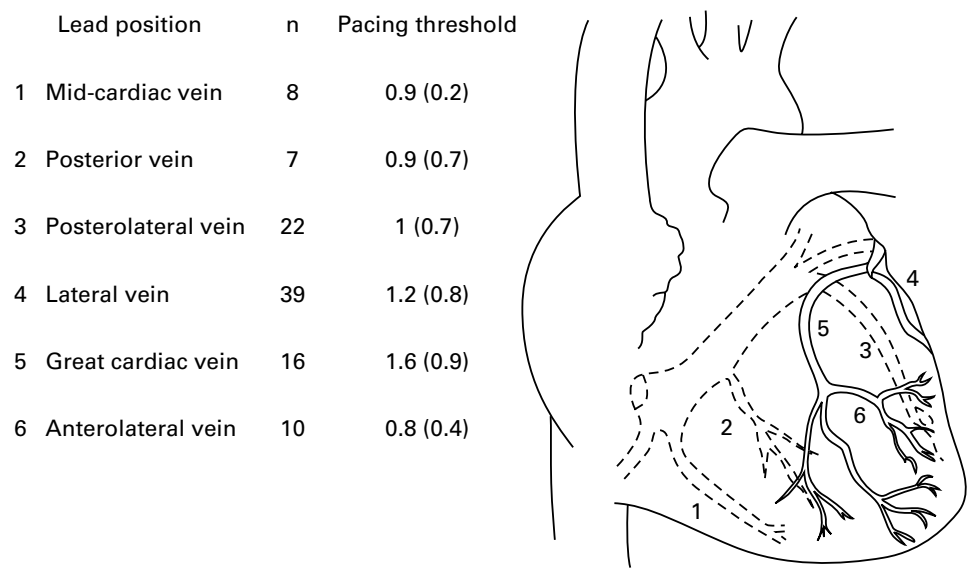

Figure 1 Left ventricular lead position and implant pacing thresholds.

\section{IMPLANTATION PROCEDURE}

Implantations were performed by two operators under local anaesthesia. All patients were premedicated (hydroxyzine and morphine chlorhydrate) and received antibiotic prophylaxis. The cephalic vein was isolated by a prepectoral incision and was used to implant the right ventricular and right atrial leads whenever possible. The left ventricular lead was inserted through the subclavian vein.

Since April 1999, we have systematically used long preshaped guiding sheaths which permit stable catheterisation of the coronary sinus, the pacing lead being subsequently introduced through that sheath. The sheath also permits selective coronary sinus angiography with a catheter fitted with an occlusive balloon. Previously, the left ventricular pacing lead would be introduced directly into the coronary sinus on the basis of anatomical and intracardiac ECG criteria. To facilitate coronary sinus catheterisation, a preshaped stylet with an approximately $45^{\circ}$ terminal angle was inserted into the lead catheter. Once inside the coronary sinus, the lead is guided into a cardiac vein. From August 1994 to January 1996, unipolar, non-specific, tine-free leads were used for left ventricular pacing. From 1996 onwards, we used leads specially designed for pacing through the coronary sinus. Initially, these were leads dedicated to left atrial pacing (Medtronic 2188, Medtronic Inc, Minneapolis, USA), and subsequently leads specially designed for left ventricular pacing through the coronary sinus were used (Medtronic 2187, and other leads under current evaluation).

Our aim was to position the left ventricular lead at the latest activation site of the left ventricle in spontaneous rhythm-that is, most often in a lateral, anterolateral, or posterolateral vein - and the right ventricular lead at the earliest activation site, so as to obtain the longest interventricular conduction time and a paced QRS duration that was as short as possible when pacing the two sites simultaneously. In practice, the left ventricular lead was implanted first.

In the absence of any accessible lateral veins, or if pacing thresholds were too high (that is, $>2.5 \mathrm{~V}$ at a $0.5 \mathrm{~ms}$ pulse width), another branch would be chosen and several right ventricular pacing sites would then be tested to obtain the best possible paced QRS during biventricular pace mapping.

In the right ventricle, a distinction was made between the following locations: apical region, pulmonary outflow tract, interventricular septum, and other locations.

In the left ventricle, six locations were individualised (fig 1): mid-cardiac vein, posterior cardiac vein, posterolateral cardiac vein, lateral vein, great cardiac vein, and anterolateral vein.

\section{PACEMAKER}

In patients who were in permanent atrial fibrillation, the left ventricular lead was connected to the atrial port of a DDDR pacemaker and the right ventricular lead to the ventricular entry, and the atrioventricular delay was programmed to its minimum value $(30 \mathrm{~ms})$. The pacing mode was therefore VVIRbiventricular. In those patients, the atrioventricular junction was systematically ablated at the time of implantation to ensure full and permanent ventricular capture.

In patients in sinus rhythm and in the early period of the experiment, both ventricular leads were connected to the ventricular port of a standard dual chamber pacemaker, using a Y bifurcated adapter. Dedicated pacemakers are now available which feature two ventricular entries (Chorum MSP, ELA médical, Le Plessis Robinson, France; InSync, Medtronic Inc, Minneapolis, USA; Contak, Guidant, St Paul, Minneapolis, USA). Pacing was delivered in biventricular DDD mode, the atrioventricular delay being optimised in each patient, based on the mitral flow morphology on Doppler echocardiography. ${ }^{11}$

\section{EVALUATION}

Patients were followed up one and three months after pacemaker implantation and every six months thereafter. Follow up consisted of: a clinical examination, functional evaluation (NYHA classification, cardiorespiratory exercise test), 12 lead surface ECG recorded at $50 \mathrm{~mm} / \mathrm{s}$, frontal and lateral chest $x$ ray for lead position analysis, pacing and sensing threshold, and lead impedance measurements through pacemaker telemetry. Finally, pacemaker related or lead related complications were listed.

\section{Results}

CLINICAL DATA

One hundred and two consecutive patients (mean (SD) age, 67 (9) years) who received biventricular pacemaker implantations between August 1994 and February 2000 were included in the study. Heart disease aetiology was ischaemic in 37 patients, idiopathic in 50, and secondary to another cause in 15. All were symptomatic and were in NYHA functional class III $(n=71)$ or IV $(n=31)$, despite optimised medical treatment. Thirty one patients $(30 \%)$ had chronic atrial fibrillation. QRS duration was 185 (26) ms on average. In 
Table 1 Evolution of clinical variables with biventricular pacing

\begin{tabular}{llll}
\hline Variable & Before implantation & After implantation & p Value \\
\hline QRS (ms) & $185(26)$ & $158(19)$ & $<0.001$ \\
QRS axis & $-19(61)^{\circ}$ & $48(78)^{\circ}$ & $<0.001$ \\
NYHA class & $3.3(0.5)$ & $2.0(0.6)$ & $<0.001$ \\
$\dot{\text { V }} \mathrm{O}_{2}$ max (ml $\left./ \mathrm{kg} / \mathrm{min}\right)$ & $12.3(4.7)$ & $16.3(4.3)$ & $<0.001$ \\
LVEF $(\%)$ & $22(6)$ & $26(8)$ & $<0.001$ \\
\hline
\end{tabular}

LVEF, left ventricular ejection fraction; NYHA, New York Heart Association functional class; $\dot{\mathrm{V}}_{2}$ max, maximum oxygen consumption.

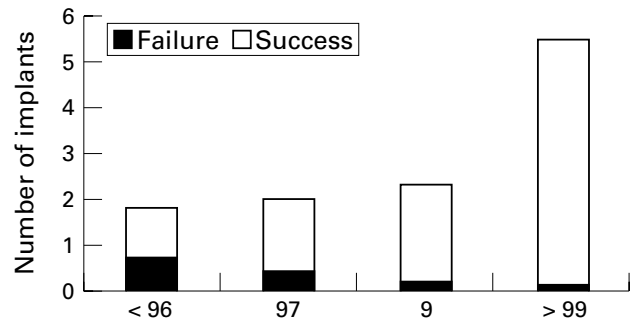

Figure 2 Change in implantation rate over time.

patients who were in sinus rhythm, the PR interval was 242 (52) ms.

The patients' characteristics before implantation and during the course of follow up are summarised in table 1 .

The patients' functional tolerance improved significantly with biventricular pacemaker implantation, with a mean reduction of 1.2 NYHA grades and a 32\% increase in peak oxygen consumption ( $\dot{\mathrm{V}}_{2}$ peak). After a mean (SD) follow up of 15 (13) months, $21 \%$ of patients had died. The one year survival rate was $90 \%$.

IMPLANTATION

Of the 116 patients eligible for biventricular pacemaker implantation, 102 had left ventricular leads implanted - an overall 88\% implantation success rate.

Operator training and the use of specific tools resulted in an increase in the success rate from $61 \%$ at the beginning of the experiment to $98 \%$ during the last year (fig 2).

Among the 102 patients with lead implantation, the left ventricular lead was positioned in a lateral, anterolateral, or posterolateral vein in 71 patients $(69 \%)$, in a posterior vein in seven, in the mid-cardiac vein in eight, and in the great cardiac vein in 16 (fig 1 ).

The right ventricular lead was positioned at the apex in 22 patients, in the outflow tract in 12 , in the septum in 47 , and elsewhere in 21 .

PERIOPERATIVE RESULTS

Biventricular pacing significantly reduced QRS duration at baseline (158 (19) ms $v 185$ (26) $\mathrm{ms}, \mathrm{p}<0.001)$. Left ventricular pacing thresholds were $1.1 \mathrm{~V}$ on average, $\mathrm{R}$ wave amplitude was $13 \mathrm{mV}$ and pacing impedance was $720 \mathrm{ohms}$. Table 2 summarises pacing threshold, intracardiac signal amplitude, and left ventricular lead impedance at implantation and during follow up.

The mean operating time was 134 (43) $\mathrm{min}$ and the mean fluoroscopy time was 45 (24) $\min$.

\section{PERIOPERATIVE COMPLICATIONS}

In the course of the 102 operations, the guide sheath caused coronary sinus dissection in two cases. This had no clinical impact and the two patients were reimplanted later, after spontaneous healing of the coronary sinus.

LEAD FOLLOW UP

In the course of a mean follow up duration of 15 (13) months, pacing threshold increased from a mean of $1.1 \mathrm{~V}$ at implantation to a mean of $1.9 \mathrm{~V}$ at the end of follow up. This increase occurred at an early stage during the first month, and pacing thresholds later remained stable. In contrast, $\mathrm{R}$ wave amplitude remained relatively stable. Pacing impedance decreased slightly during follow up (table 2). No significant differences were observed in the evolution of lead impedance during follow up when the Y adapter connection was compared with independent connection of the two ventricular leads to separated entry points (527 (320) and 649 (308) ohms, respectively, at the end of follow up).

\section{REOPERATIONS}

Twenty six reoperations (25\%) were necessary within a period of two days to 48 months after the initial implantation. In most cases, reoperation was required because of a sharp increase in left ventricular pacing threshold $(n=15)$. These threshold increases were linked either to dislocation of the lead or to a connector defect. In three patients, phrenic nerve stimulation occurred which required the left ventricular lead to be repositioned. We systematically looked for phrenic nerve stimulation at the time of implantation by programming pacing output at $10 \mathrm{~V}$. When phrenic nerve stimulation was detected we changed the lead position. This probably explains the low incidence of phrenic nerve stimulation in our population.

In seven cases, reoperation was needed because of an increase in right ventricular pacing threshold or because of right ventricular lead dislocation. The rate of reoperation for lead dislodgement also decreased with time, from $30 \%$ at the beginning of the experiment to $11 \%$ since 1999 .

Table 2 Evolution of left ventricular lead electrical variables during follow up

\begin{tabular}{|c|c|c|c|c|c|c|c|c|c|}
\hline \multirow[b]{2}{*}{ Type of LV lead } & \multicolumn{3}{|c|}{ Time of implantation } & \multicolumn{3}{|l|}{ Month 3} & \multicolumn{3}{|c|}{ End of follow up } \\
\hline & $T(V)$ & $R(m V)$ & $I(\Omega)$ & $T(V)$ & $R(m V)$ & $I(\Omega)$ & $T(V)$ & $R(m V)$ & $I(\Omega)$ \\
\hline Medtronic 2188 & $1.4(0.9)$ & $12.5(6.0)$ & $681(216)$ & $1.7(0.8)$ & $14.0(6.0)$ & $624(245)$ & $2.0(1.0)$ & $10.5(5.0)$ & $637(388)$ \\
\hline Medtronic 2187 & $0.9(0.7)$ & $14.0(8.0)$ & $739(184)$ & $1.7(0.7)$ & $9.0(4.0)$ & $492(150)$ & $1.9(0.9)$ & $10.0(7.0)$ & $567(242)$ \\
\hline Recent leads & $0.9(0.4)$ & $12(5.5)$ & 749 (171) & $1.5(0.8)$ & $7.2(3.7)$ & 445 (117) & NA & NA & NA \\
\hline Old leads & $1.1(0.9)$ & $14.6(6.0)$ & $705(113)$ & $2.3(0.2)$ & $13.0(2.0)$ & $429(73)$ & $1.6(0.7)$ & $16.0(4.0)$ & $434(121)$ \\
\hline All & $1.1(0.7)$ & $13.0(7.0)$ & $720(190)$ & $1.7(0.7)$ & $11.0(5.0)$ & $526(193)$ & $1.9(0.9)$ & $11.0(5.0)$ & $600(332)$ \\
\hline
\end{tabular}

Values are mean (SD)

$\mathrm{I}(\Omega)$, pacing impedance (ohms); R (mV), R wave amplitude (mV); $\mathrm{T}(\mathrm{V})$, pacing threshold (volts). 
Finally, three patients developed infection in the pacing system, resulting in the need for explantation. These infectious complications occurred 10 days, 21 days, and three months after implantation.

In 15 cases it was necessary to remove the left ventricular lead, either because of infection or to relocate it or replace it with another one. Eight such extractions were performed. In all cases the lead had been in position for more than one month, the oldest having been in place for 41 months. In all cases it was possible to remove the left ventricular lead from the cardiac vein by simple traction with no difficulty. There were no complications from this procedure. Finally, coronary sinus angiography was performed in three patients after the left ventricular lead was removed. This showed no thrombosis, stenosis, or coronary sinus dissection.

During the follow up period, no reintervention was necessary for premature battery depletion.

\section{Discussion}

It appears from our experience that biventricular pacing is a safe technique. Indeed, no life threatening complications were observed during the course of the study. The two coronary sinus dissections - which occurred during pacemaker implantation-had no consequences for the patients. Spontaneous healing of the coronary sinus allowed successful later implantation of a left ventricular lead in these patients. With regard to infectious complications, the rate in this study was 3\%-that is, slightly higher than described for standard single or dual chamber pacemaker implantation. ${ }^{12}$ Infections occurred early after the procedure, probably related to the long duration of the operation.

Our study also shows that left ventricular pacing using a lead placed in a coronary sinus tributary has a good success rate, though the

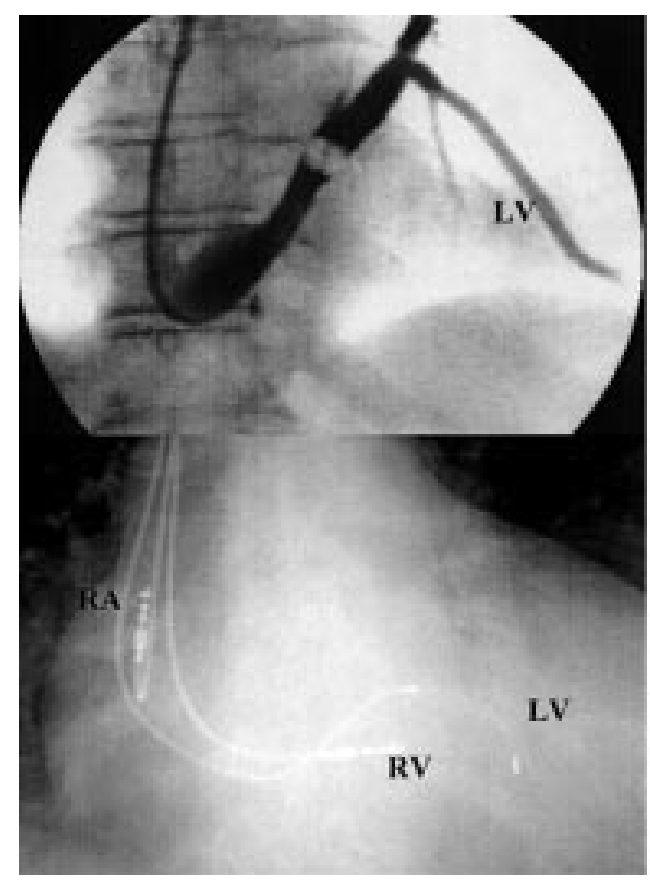

requirement for reoperation was relatively high at $25 \%$. Nevertheless it should be stressed that the success rate has increased and the reoperation rate linked to lead displacement has decreased with increasing experience with the technique. This improvement in outcome is probably linked to the learning curve of the implantation operators, and also to the availability of new equipment. As seen in fig 2, the success rate has increased dramatically in parallel with the overall number of implantations, from $61 \%$ at the beginning of the study to $98 \%$ in the most recent year.

Both the use of preshaped sheaths that permit stable coronary sinus catheterisation and the advent of selective angiography of the coronary sinus and branches have contributed to the increased success rate of pacemaker implantation. ${ }^{13}$ The saving in time that has resulted from these innovations is valuable in the light of the large anatomical differences in the coronary sinus and its branches from one patient to another. ${ }^{14}$

The introduction of new leads has also reduced the number of failures. Smaller diameter leads facilitate coronary vein catheterisation and allow easier passage around tortuous vessels. They also ensure better contact and hence improved electrical properties. Some investigators have stressed the value of new leads featuring a side wire or over-the-wire technology - similar to that used in coronary angioplasty - to facilitate access to the coronary veins. ${ }^{15}$

The development of new equipment has thus made it possible to increase the success rate and reduce operation time, but does it ensure optimal pacing configuration?

Most teams consider that the optimal position of the left ventricular lead is in the lateral wall of the left ventricle. Some investigators have indeed reported that in dilated cardiomyopathies with conduction disorders the left

Figure 3 In this example there is a large lateral vein in which a thick conventional lead could easily be introduced. $L V$, lateral vein; $R A$, right atrial lead; $R V$, right ventricular lead; LV, left ventricular lead.

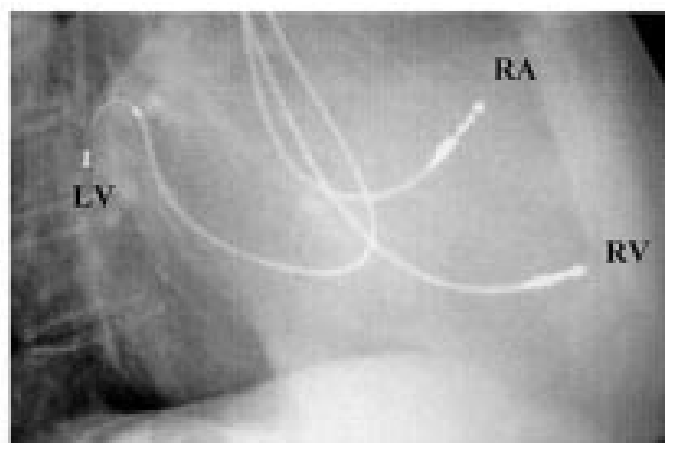



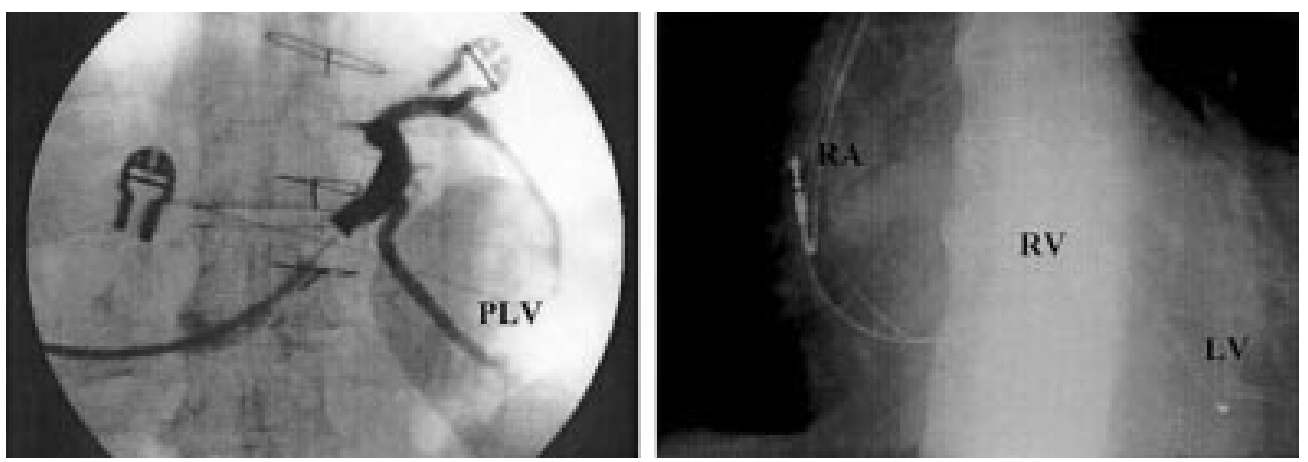

Figure 4 In this coronary sinus angiogram (left) there was a large posterolateral vein. However, the angle it makes with the coronary sinus may make it difficult to reach. In this case a very thin lead, with side wire technology, could be introduced into the vein (chest $x$ ray on the right). LV, left ventricular lead; PLV, posterolateral vein; RA, right atrial lead; $R$, right ventricular lead.
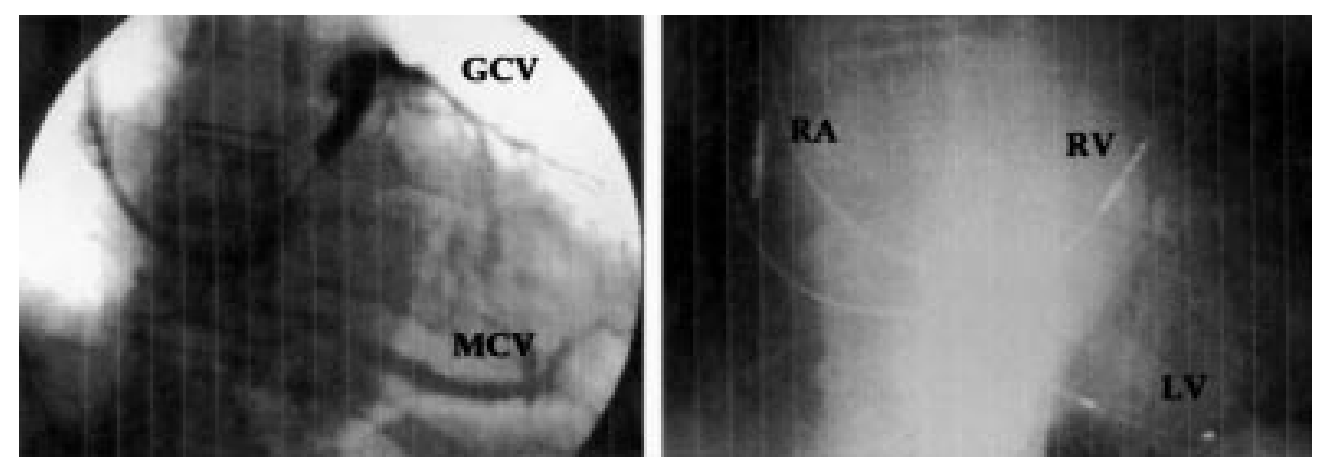

Figure 5 It can be seen on this coronary sinus angiogram (left) that there was no lateral or posterolateral vein, so that a choice had to be made between the great cardiac vein and the mid-cardiac vein. In this case the left ventricular lead was placed in the mid-cardiac vein, as can be seen on the chest $x$ ray (right). GCV, great cardiac vein; LV, left ventricular lead; $M C V$, mid-cardiac vein; $R A$, right atrial lead; $R V$, right ventricular lead.

ventricular lateral wall often shows delayed contraction. ${ }^{16}$ After analysing the acute haemodynamic effects of $\mathrm{BiV}$ pacing, others have concluded that the best configuration includes pacing of the left ventricular free wall. ${ }^{17}$ However, achieving the optimum configuration is subject to certain limitations linked to the anatomical variability of the coronary sinus and its branches. Indeed, even though the mid- and great cardiac veins are constantly present, no lateral or posterior branches can be found in around $1 \%$ of patients. ${ }^{14}$ In addition, the diameter of the branches and the angles they make with the coronary sinus also vary between patients, making these vessels hard to access. In figs 3,4 , and 5 we show the anatomical variability of the coronary sinus and branches. Figure 3 is an example where the gauge and angle made by the coronary sinus with a lateral branch make the branch readily accessible to a pacing lead. In fig 4, however, angiography shows the presence of a good calibre posterolateral branch, but its angle with the coronary sinus may create difficulty in introducing a lead. Leads featuring angioplasty-type guidewires here prove their worth. Finally, in the example shown in fig 5 there is no lateral or posterior branch. In that particular case, a choice had to be made between the great cardiac vein, which is small in diameter, and the mid-cardiac vein. In our study, $70 \%$ of left ventricular leads were positioned in a lateral, anterolateral, or posterolateral branches of the coronary sinus. The lack of a lateral branch or the presence of a high pacing threshold forced us to select other locations in some cases.

It should be noted that pacing and sensing electrical properties did not vary significantly with the position of the lead (fig 1). Electrical resynchronisation also appeared to be satisfactory, because QRS duration was reduced by $15 \%$ on average and the QRS axis tended to normalise.

The pacing mode induced a significant functional improvement in our patients, as reflected by the downgrading of the NYHA classification (by one class on average), improved exercise tolerance, and an increase in average $\dot{\mathrm{V}}_{2}$ peak of $32 \%$.

\section{CONCLUSIONS}

Transvenous biventricular pacing is a feasible technique with a low complication rate. There have been no serious complications in our patients. An initially high rate of implantation failure because of difficulties with the left ventricular lead has decreased greatly with the increasing experience of the operators and with the development and introduction of new leads and equipment. Further improvements are likely, in parallel with ongoing technological advances.

1 Cazeau S, Ritter P, Bachdach S, et al. Four chamber pacing in dilated cardiomyopathy. Pacing Clin Electrophysiol 4;17:1974-9.

2 Bakker PF, Meijburg H, de Vries J, et al. Biventricular pacing in end-stage heart failure improves functional capacity and left ventricular function. F Intervent Cardiol Electrophysiol 2000;4:395-404. 
3 Leclercq C, Cazeau S, Le Breton H, et al. Acute hemodynamic effects of biventricular DDD pacing in patients with end-stage heart failure. $\mathcal{F} \mathrm{Am}$ Coll Cardiol

4 Kass D, Chen CH, Curry C, et al. Improved left ventricular mechanics from acute VDD pacing in patients with dilated cardiomyopathy and ventricular conduction delay. Circulation 1999;99:1567-73.

5 Blanc JJ, Etienne Y, Gilard M, et al. Evaluation of different ventricular pacing sites in patients with severe heart failure. Results of an acute hemodynamic study. Circulation 1997;96:3273-7.

6 Auricchio A, Stellbrink C, Block M, et al. Effects of pacing chamber and atrioventricular delay on acute systolic function of paced patients with congestive heart failure. Circulation 1999;99:2993-3001.

7 Gras D, Mabo P, Tang T, et al. Multisite pacing as a supplemental treatment of congestive heart failure: preliminary results of the Medtronic Inc InSync study. Pacing Clin Electrophysiol 1998;21:2249-55.

8 Daubert JC, Cazeau S, Leclercq C. Do we have reasons to be enthusiastic about pacing to treat advanced heart be enthusiastic about pacing to treat
failure? Eur 7 Heart Failure 1999;1:281-7.

9 Jais P, Douard H, Shah DC, et al. Endocardial biventricular pacing. Pacing Clin Electrophysiol 1998;21:2128-31.
10 Daubert JC, Ritter P, Le Breton H, et al. Permanent left ventricular pacing with transvenous leads inserted into the coronary veins. Pacing Clin Electrophysiol 1998,21:239-45.

11 Kindermann M, Frohlig G, Doerr T, et al. Optimizing the AV delay in DDD pacemaker patients with high degree AV block: mitral valve Doppler versus impedance cardiography. Pacing Clin Electrophysiol 1997;20:2453-62.

12 Kiviniemi MS, Pirnes MA, Eranen HJ, et al. Complications related to permanent pacemaker therapy. Pacing Clin Electrophysiol 1999;22:711-20.

13 Blanc JJ, Benditt D, Gilard M, et al. A method for permanent transvenous left ventricular pacing. Pacing Clin Electrophysiol 1998;21:2021-4.

14 Gilard M, Mansourati J, Etienne Y, et al. Angiographic anatomy of the coronary sinus and its tributaries. Pacing Clin Electrophysiol 1998;21:2280-4.

15 Walker S, Levy T, Paul R, et al. The use of a "side wire" permanent transvenous pacing electrode for left ventricular manent transvenous pacing electro

16 Rosenbush S, Ruggie N, Turner D, et al. Sequence and timing of ventricular wall motion in patients with bundle ing of ventricular wall motion in patients

17 Auricchio A, Klein H, Tockman B, et al. Transvenous biventricular pacing for heart failure: can the obstacles be overcome? Am f Cardiol 1999;83:136-42D.

\section{IMAGES IN CARDIOLOGY}

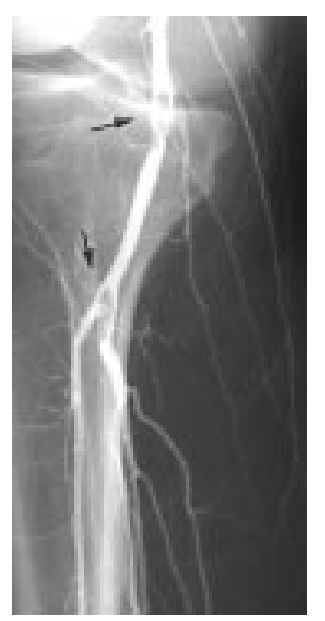

\section{Aortic recoarctation as the source of arterial embolism 32 years after synthetic patch angioplasty}

A 44 year old man presented with intermittent claudication of the right lower leg and a history of surgical repair for aortic coarctation at the age of 12 years. Right popliteal and pedal pulses were diminished, and a 3/6 mesosystolic heart murmur was most clearly audible over the posterior part of the thorax. Systolic blood pressure was slightly raised equally in both arms, and a systolic ankle/arm pressure gradient that was higher on the right than on the left side $(75 \mathrm{~mm} \mathrm{Hg}$ and $45 \mathrm{~mm} \mathrm{Hg}$, respectively) was found. Ultrasound showed a mass in the right popliteal artery causing severe

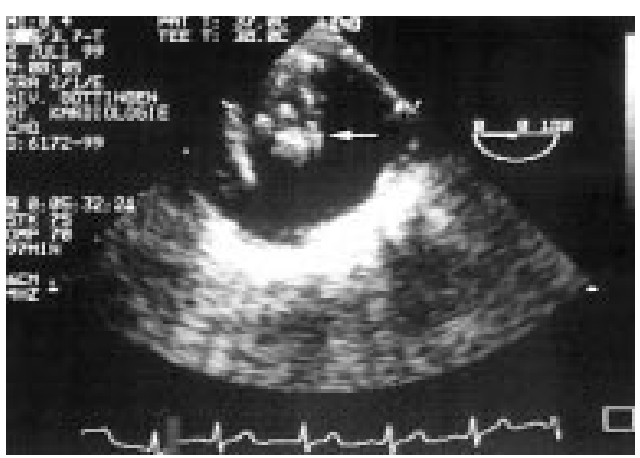

stenosis. Angiography revealed two structures in the middle and just before the bifurcation of the popliteal artery (far left). Based on these findings, a subacute popliteal artery embolism was suspected. No irregularities were found in the lower aorta and iliac/femoral arteries. Other causes of the embolism, such as atrial fibrillation, endocarditis or patent foramen ovale, were excluded.

Transoesophageal echocardiography showed high grade aortic recoarctation with an extensively protruding and partly mobile sclerotic structure (left) at the site where the patient had undergone corrective surgery with Dacron patch angioplasty 32 years previously. Resection of the restenosed aortic segment with interposition of a synthetic vascular tube graft was performed. The preoperative systolic pressure gradient of $40-50 \mathrm{~mm} \mathrm{Hg}$ greatly improved, but intermittent claudication remained unchanged. The claudication was resolved only after subsequent balloon angioplasty of the right popliteal artery.

DIRK VOLLMANN WOLGANG RUSCHEWSKI CHRISTINA UNTERBERG dirkvolmann2000@aol.com 